\title{
TALIP Perspectives, Guest Editorial Commentary
}

\section{What Counts (and What Ought to Count)?}

\author{
KENNETH CHURCH, IBM
}

We agree on Publish or Perish, but do conference papers count as publications? How about articles in new journals (that aren't yet "indexed")?

The Association for Computational Linguistics (ACL) recently started a second journal. ${ }^{1}$ One (of many) reasons for starting a second journal was a concern that some of us were hearing from our colleagues in Asia (and elsewhere) that, while conferences may count for publication in top universities in the United States, most universities have different rules. This is a serious problem for societies like the ACL: Basically, the vast majority of publications don't count. According to the ACL Anthology Network (AAN) [Radev et al. 2009, 2013] $]^{2}$, the ACL has published 14k conference papers and just 750 journal articles. We need to make room for more journal articles or else researchers in our field won't be promoted as much as researchers in other fields.

Citations are also skewed strongly toward conferences, though not as much as publications. The AAN reports $82 \mathrm{k}$ citations in ACL conference papers and $13 \mathrm{k}$ citations in ACL journals. The fact that conferences account for more publications than citations

$$
\frac{14 k}{14 k+750} \approx 95 \%>>\frac{82 k}{82 k+13 k} \approx 86 \%
$$

suggests that journal publications ought to count more. Most researchers agree that journals should count more, but how much more? Should conference papers count a little, or not at all? ${ }^{3}$

Google Scholar Citations is establishing a new standard. Their list of my top 20 publications $^{4}$ includes 9 journal articles and 11 conference papers. Under the rules found in most universities around the world, the majority of my top 20 papers don't count. I'm glad that my career never depended on those rules, but for people in our field who do have to live under those rules, we must continue to do what we can to make room for more journal publications.

Actually, the rules are even worse. Conferences often don't count, but journals also don't count-until the journal is "indexed." Unfortunately, the indexing process is tainted by an obvious profit motive. There is a credible concern that for-profit indexing services might favor for-profit journals. In fact, when for-profit publishers are competing for a new journal, one of their selling points is their track record in advocating new journals to the for-profit indexing services with the implication that non-profit publishers (ACL, ACM, and IEEE) are relatively less successful in adding new journals to the indexes as quickly.

There is a need for someone to do a careful comparison of Google Scholar with Web of Science and see which indexing service agrees more with surveys of experts. Which is

\footnotetext{
${ }^{1}$ http://www.transacl.org/

${ }^{2} \mathrm{http} / / /$ clair.eecs.umich.edu/aan

${ }^{3}$ See Bowyer [2012] and references therein for excellent mentoring advice to junior faculty on whether to publish in conferences or journals.

${ }^{4} \mathrm{http}: / /$ scholar.google.com/citations?user=E6aqGvYAAAAJ
}

(C) 2014 ACM 1530-0226/2014/02-ART5 $\$ 15.00$

DOI : http://dx.doi.org/10.1145/2559789 
better at predicting expert opinion on questions such as ranking of papers, researchers, venues, institutions, etc.? Can we come up with an empirical justification for indexing some journals and excluding others as commercial indexes do, or is it better to treat the world as flat as Google does? ${ }^{5}$ Hopefully, the rules in universities around the world will eventually converge toward indexes that agree with expert opinion and will not be too influenced by other considerations such as the perception and/or reality of profit motives.

Many of the criticisms of Google Scholar are similar to the criticisms that we used to hear about Wikipedia. Not so long ago, the encyclopedia market was dominated by for-profit publishers. No one could imagine, back then, that the wisdom of the crowd could compete with experts on accuracy, but these days, there is more respect for "free." The New York Times reported that Encyclopaedia Britannica went out of print in 2010, citing competition with Wikipedia, as well as a study in Nature [Giles 2005] that failed to find a difference in accuracy. ${ }^{6}$ Just as "free" changed the encylopedia business, "free" indexing services like Google Scholar are changing the "professor business." Sooner or later, the wisdom of the crowd will decide what counts and who gets promoted.

\section{H-INDEX INFLATION}

As impressive as Google Scholar is, care needs to be taken when making certain comparisons. H-index not only measures something about authors, but it also measures something about the community. It is not surprising that $\mathrm{h}$-index favors senior researchers with long careers, but h-index inflation is less well known. We observe that h-index increases over time, long after the author stops publishing.

$\mathrm{H}$-indexes are reported in a number of influential places such as Palsberg's list of computer science researchers with $h>40 .^{7}$ When Palsberg started his list, 40 was more exclusive than it is today (because of inflation).

The definition of h-index sounds simple enough to compute: $h$ is the number of papers with $h$ or more citations [Hirsch 2005]. The problem is that standard citation resources are far from perfect. Standard resources such as Google Scholar and Thomson Reuters Web of Science do not always make it clear who is citing what and who wrote what. Common/confusable names such as "John Smith" and "Ping Li" are difficult to resolve, especially since some citation formats encourage the use of initials instead of first names. Palsberg ${ }^{8}$ recommends a number of easy-to-use interfaces for computing an h-index including scHolar index, ${ }^{9}$ QuadSearch, ${ }^{10}$ and Harzing's publish or perish. ${ }^{11}$ It is even easier to estimate h-index when authors do much of the heavy lifting and use a tool such as Google Scholar Citations to make it clear what they wrote and what they didn't write.

Because the computation of h-index often requires considerable manual effort, especially for authors with common/confusable names, it isn't feasible to recompute estimates as often as we should. Since there is considerable inflation in Figure 1, which

\footnotetext{
${ }^{5}$ Freyne et al. [2010] compares an impact factor based on Google Scholar with ISI Web of Knowledge and finds that both of them predict similar rankings of conferences and journals. For this ranking task, it probably doesn't matter all that much whether we take Google's more inclusive view or a more exclusive view practiced by commercial indexes, but for more challenging ranking tasks such as ranking researchers, the inclusive/exclusive distinction is probably more important.

${ }^{6} \mathrm{http} / / /$ mediadecoder.blogs.nytimes.com/2012/03/13/after-244-years-encyclopaedia-britannica-stops-the-presses

${ }^{7}$ http://www.cs.ucla.edu/ palsberg/h-number.html

${ }^{8}$ http://www.cs.ucla.edu/ palsberg/h-number.html

${ }^{9} \mathrm{http} / / /$ interaction.lille.inria.fr/ roussel/projects/scholarindex

10 http://quadsearch.csd.auth.gr/

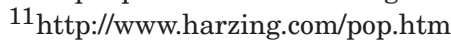




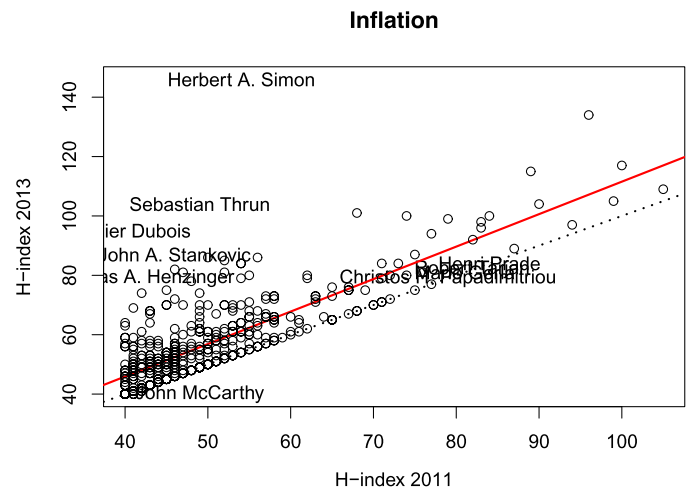

Fig. 1. H-indexes generally increase over time (most points are above the dashed black line $(x=y)$ ). The scatterplot compares h-indexes from 2011 with h-indexes from 2013 for 480 authors as reported on http://www.cs.ucla.edu/ palsberg/h-number.html. The solid red line shows the regression line: $h 2013 \approx$ $2.16+1.09 \times h 2011$, suggesting an inflation rate of at least $9 \%$.

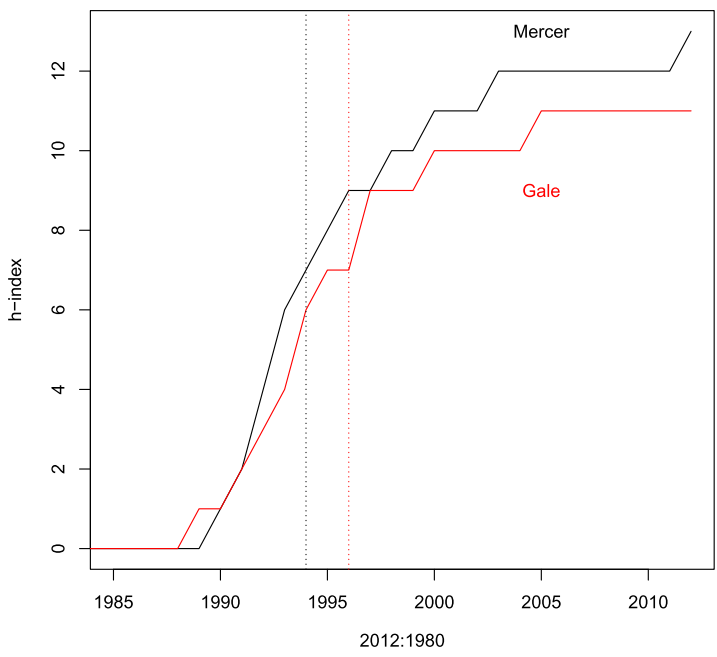

Fig. 2. This plot drills down into two authors in Figure 3, Bob Mercer (black) and Bill Gale (red). Note that h-index continues to increase well after they stopped publishing in the mid-1990s (dashed lines). Gale's h-index score continued to increase well after his death in 2002.

compares Palsberg's current values with historical estimates ${ }^{12}$, these scores need to be updated annually. Stale estimates for one researcher should not be compared with fresh estimates for another. If we recomputed h-indices annually, as we should, then fewer points would fall on the $x=y$ dashed line in Figure 1 . H-index is unlikely to be flat over two years even for an author that hasn't published in a decade, because, for example, they left the field or passed away (Figure 2).

Since the ACL Anthology is so much smaller than Google Scholar, it has been possible for the authors of the ACL Anthology Network to clean up the citation graph so there is much less question about who cites whom and who wrote what when. As a

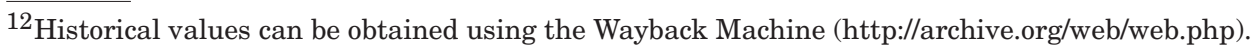




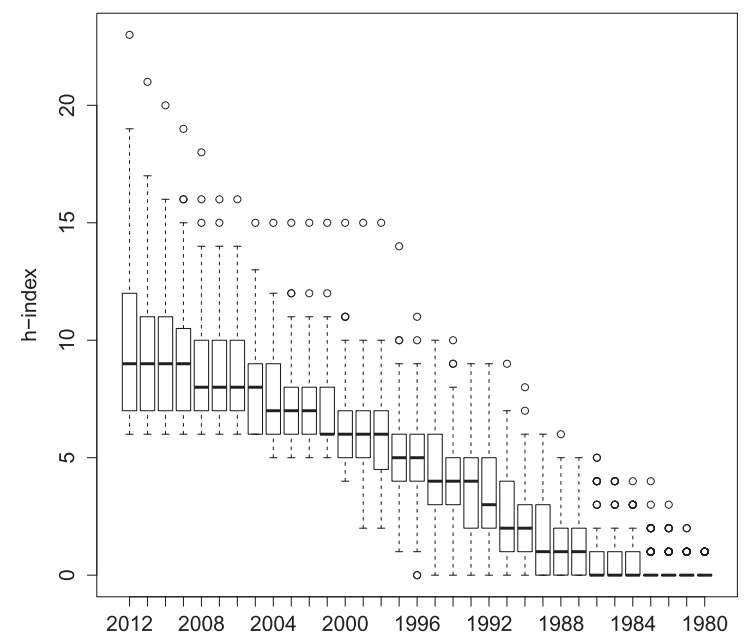

Fig. 3. For the ACL Anthology Network, it is possible to estimate h-index scores over all time. The plot shows h-indices for the top 100 authors (in 2001). Scores have nearly doubled since then.

consequence, it was relatively easy to compute h-index estimates for all authors over all time (Figure 3).

Care needs to be taken when making certain comparisons. Since the Anthology excludes non-ACL publications, h-index scores from the Anthology tend to be smaller than Google's scores. In general, h-index scores tend to be larger when the index is larger and more inclusive. H-index scores based on Google Scholar tend to be larger than scores based on smaller, more exclusive indices such as the ACL Antholgy and Thomson Reuters Web of Science. It would be a mistake to compare scores from one source with scores from another source without calibrating for the differences in the two sources. Similarly, as already mentioned, because of inflation, it would be a mistake to compare stale estimates for one researcher with fresh estimates for another.

\section{CONCLUSIONS}

Despite potential pitfalls such as those mentioned, measures such as h-index based on freely available publication data will likely, in time, supersede more traditional (for-profit) indices. And this will lead, one hopes, to a view of publications that ranks researchers, papers, venues, institutions, etc. based more on their contributions to the field over time and less on publishers and venues (and commercial concerns). In the meantime, though, we need to continue to expand avenues for journal publication in our field and do what we can to make sure that as many of our journals as possible (including ACM's TALIP and ACL's TACL) are indexed in as many indices as possible.

\section{REFERENCES}

Kevin W. Bowyer. 2012. Mentoring advice on "Conferences Versus Journals" for CSE faculty. Bibsonomy. http://nd.edu/ kwb/Mentoring_Conferences_Journals.pdf.

Jill Freyne, Lorcan Coyle, Barry Smyth, and Padraig Cunningham. 2010. Relative status of journal and conference publications in computer science. Commun. ACM 53, 11, 124-132. DOI : http://dx.doi.org/10.1145/1839676.1839701.

Jim Giles. 2005. Internet encyclopaedias go head to head. Nature 438, 900-901. DOI : http://dx.doi.org/10.1038/438900a. 
Jorge E. Hirsch. 2005. An index to quantify an individual's scientific research output. In Proceedings of the National Academy of Sciences of the United States of America 102, 46, 16569-16572.

Dragomir R. Radev, Pradeep Muthukrishnan, and Vahed Qazvinian. 2009. The ACL anthology network corpus. In Proceedings of the ACL Workshop on Natural Language Processing and Information Retrieval for Digital Libraries. ACL, Singapore, 54-61.

Dragomir R. Radev, Pradeep Muthukrishnan, Vahed Qazvinian, and Amjad Abu-Jbara. 2013. The ACL anthology network corpus. Language Resources and Evaluation, 1-26. DOI : http://dx.doi.org/10.1007/s10579-012-9211-2. 